\title{
Gut microbiome affects the response to anti-PD-1 immunotherapy in patients with hepatocellular carcinoma
}

\author{
Yi Zheng ${ }^{1 \dagger}$, Tingting Wang ${ }^{2 \dagger}$, Xiaoxuan Tu' ${ }^{1}$, Yun Huang ${ }^{2}$, Hangyu Zhang ${ }^{1}$, Di Tan², Weiqin Jiang ${ }^{1}$, Shunfeng Cai ${ }^{2}$, \\ Peng Zhao ${ }^{1}$, Ruixue Song ${ }^{2}$, Peilu Li ${ }^{2}$, Nan Qin ${ }^{2,3^{*}}$ and Weijia Fang ${ }^{1,4,5,6^{*}}$ (D)
}

\begin{abstract}
Background: Checkpoint-blockade immunotherapy targeting programmed cell death protein 1 (PD-1) has recently shown promising efficacy in hepatocellular carcinoma (HCC). However, the factors affecting and predicting the response to anti-PD-1 immunotherapy in HCC are still unclear. Herein, we report the dynamic variation characteristics and specificities of the gut microbiome during anti-PD-1 immunotherapy in HCC using metagenomic sequencing.

Results: Fecal samples from patients responding to immunotherapy showed higher taxa richness and more gene counts than those of non-responders. For dynamic analysis during anti-PD-1 immunotherapy, the dissimilarity of beta diversity became prominent across patients as early as Week 6. In non-responders, Proteobacteria increased from Week 3, and became predominant at Week 12. Twenty responder-enriched species, including Akkermansia muciniphila and Ruminococcaceae spp., were further identified. The related functional genes and metabolic pathway analysis, such as carbohydrate metabolism and methanogenesis, verified the potential bioactivities of responderenriched species.

Conclusions: Gut microbiome may have a critical impact on the responses of HCC patients treated with anti-PD-1 immunotherapy. The dynamic variation characteristics of the gut microbiome may provide early predictions of the outcomes of immunotherapy in HCC, which is critical for disease-monitoring and treatment decision-making.
\end{abstract}

Keywords: Gut microbiome, Hepatocellular carcinoma, Anti-PD-1 immunotherapy

\section{Introduction}

With a high malignancy and a poor prognosis, hepatocellular carcinoma (HCC) is ranked as the fourth-leading cause of cancer-related fatalities worldwide, bringing a heavy burden to public health [1]. The oral multi-kinase inhibitor, sorafenib, is the standard systemic therapeutic option for advanced-stage $\mathrm{HCC}$ and has an objective response rate (ORR) of less than 5\% [2]. Recently, checkpoint blockade immunotherapy targeting programmed cell death protein 1 (PD-1) has shown promising efficacy for treating HCC. In two multicenter, phase 2 studies [3, 4] investigating the

\footnotetext{
* Correspondence: qinnan001@126.com; weijiafang@zju.edu.cn

${ }^{+} Y i$ Zheng and Tingting Wang contributed equally to this work.

${ }^{2}$ Realbio Genomics Institute, 138 Xinjunhuan Road, Shanghai, China

${ }^{1}$ Cancer Biotherapy Center, First Affiliated Hospital, School of Medicine,

Zhejiang University, 79 Qingchun Road, Hangzhou, China

Full list of author information is available at the end of the article
}

efficacy of anti-PD-1 immunotherapy in sorafenibrefractory HCC, the ORR was nearly $20 \%$, which quadrupled that of sorafenib. However, the factors affecting and predicting the response to anti-PD-1 immunotherapy in HCC are still unclear.

The role of the gut microbiome in modulating tumor responses to immunotherapy in melanoma [5-7], nonsmall cell lung cancer, renal cell carcinoma, and urothelial carcinoma $[6,8]$ has received increased attention across a series of studies in recent years. However, data concerning the impact of the gut microbiome on HCC immunotherapy have not been reported. Additionally, previous research to date has tended to focus more on the baseline status rather than a dynamic variation of the gut microbiome during immunotherapy. The aim of the present study, using fecal metagenomics as a 
snapshot, was to provide more specific understandings on how the gut microbiome influences the responses of HCC patients to anti-PD-1 immunotherapy.

\section{Results and discussion}

Eight HCC patients with Barcelona Clinic Liver Cancer (BCLC) Stage C disease treated with anti-PD-1 antibodies after progression on sorafenib were enrolled in the present study. Anti-PD-1 antibodies were administered every 3 weeks. During the treatment, no antibiotics were applied. Patients were classified as responders ( $R$, complete or partial response, or stable disease lasting for over 6 months; $n=3$ ) and non-responders (NR, progressed disease or stable disease lasting less than 6 months; $n=5$ ) based on radiological evaluation according to Response Evaluation Criteria in Solid Tumors (RECIST 1.1). Fecal samples were collected at the baseline (Day 0), Week 1 after treatment initiation, and every 3 weeks during therapy until disease progression, according to the informed consent and study protocol. Dynamic variation of gut bacterial characteristics was evaluated and analyzed by metagenomic sequencing.
Over the entire treatment, $\mathrm{R}$ showed higher taxa richness and more gene counts than those of NR (Fig. 1a). As for dynamic diversity analysis, the beta diversity evaluated by Bray-Curtis distances showed that the intergroup dissimilarity became significantly higher than the intra-group differentiation as early as Week 6 (Fig. 1b and Additional file 1: Figure S1). Dynamic microbial composition variation was also analyzed. Before treatment initiation, the Gram-positive Firmicutes, Gramnegative Bacteroidetes and Gram-negative Proteobacteria dominated the fecal microbiome of both $\mathrm{R}$ and NR, which was in accordance with the findings in healthy adults [9], suggesting that no severe gut microbiome dysbiosis was present in the study group at baseline. Specifically, Bacteroidetes were most abundant, followed by Firmicutes and Proteobacteria. As treatment proceeded, microbial composition at the phylum level in $\mathrm{R}$ remained relatively stable. However, in NR, Proteobacteria markedly increased as early as Week 3 , and became predominant at Week 12 (Fig. 1c). Increase of Proteobacteria in NR was mainly attributed to the prevalence of Escherichia coli, while the most notable proteobacterial member in $\mathrm{R}$ was Klebsiella pneumoniae. Composition

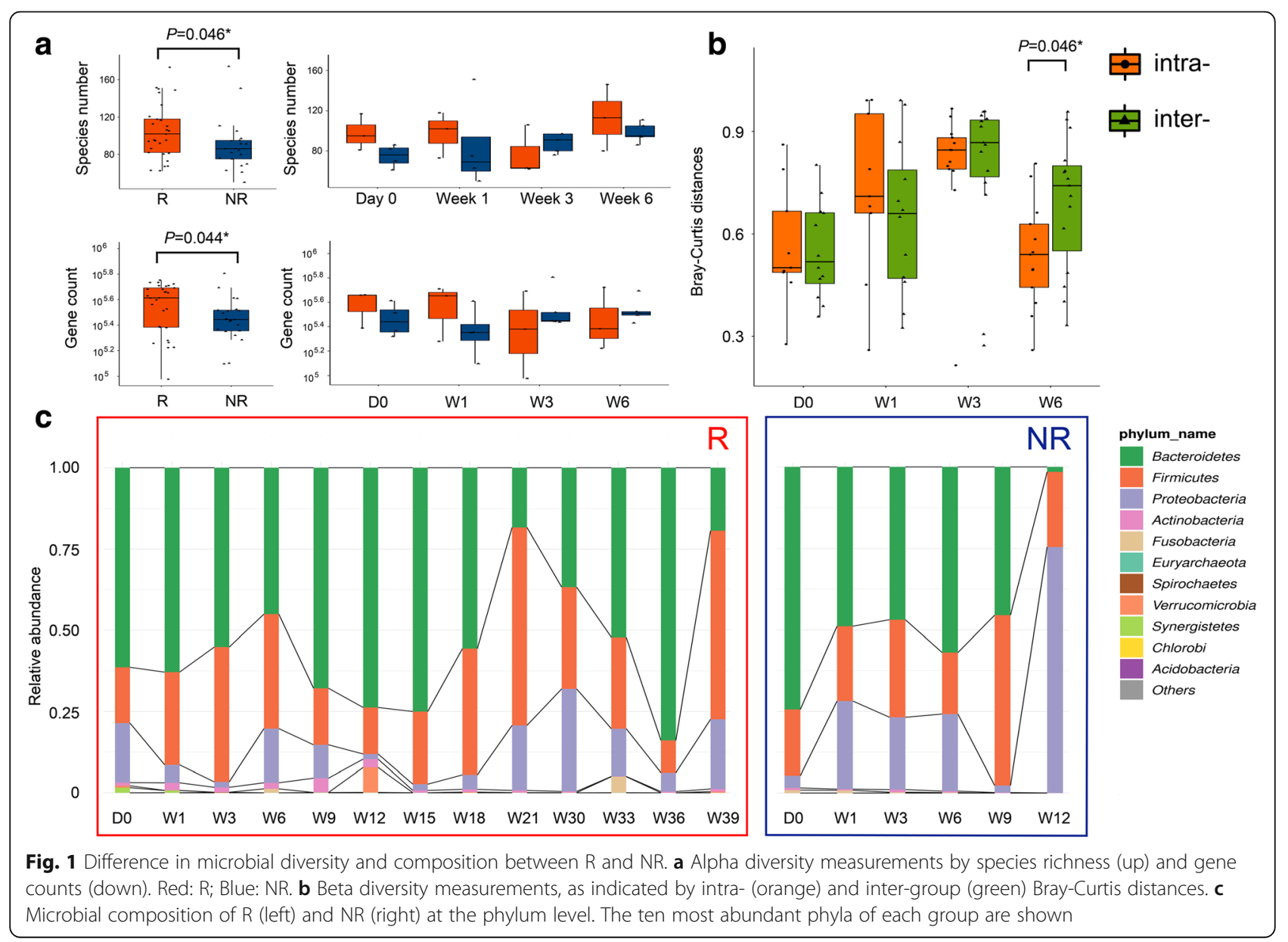


of Bacteroides and Firmicutes also exhibited individual patterns (Additional file 2: Figure S2 and Additional file 3: Figure S3). These findings suggested that the dynamic variation characteristics of gut-microbial diversity and composition at the early treatment period of anti-PD-1 immunotherapy in HCC may have distinct implications on drug efficacy and disease prognosis.

To further identify the species possibly affecting patient responses, linear discriminant analysis (LDA)-effect size (LEfSe) algorithm analysis was performed between all $\mathrm{R}$ and NR samples. Twenty R-enriched species and fifteen NR-enriched species were identified (Fig. 2a and Additional file 4: Figure S4). Among the R-enriched species, four Lactobacillus species (L. oris, L. mucosae, $L$. gasseri, and L. vaginalis), Bifidobacterium dentium and Streptococcus thermophilus were probiotic lactic acid bacteria, which were beneficial to host metabolism and immunity by inhibiting the growth of pathogenic microorganisms and accompanying spoilage agents. Oral administration of Bifidobacterium could improve tumor control efficacy of programmed cell death protein 1 ligand 1 (PD-L1)-specific antibody therapy [10]; Coprococcus comes, Bacteroides cellulosilyticus, and Subdoligranulum sp. also possessed probiotic potential, as they were reported to be related with dietary fiber digestion and short-chain fatty acid production. Notably, enrichment of one Lachnospiraceae and two Ruminococcaceae species (Lachnospiraceae bacterium 7_1_58FAA, Ruminococcus obeum, Ruminococcus bromii) and Akkermansia muciniphila in $\mathrm{R}$ was also observed. Commensal A.muciniphila and Ruminococcaceae benefited host health by preventing increases in intestinal permeability and systemic immunosuppression. In previous studies, a significantly higher relative abundance of Ruminococcaceae was identified in melanoma patients responding to anti-PD-1 immunotherapy [7], and oral supplementation with A. muciniphila could restore the

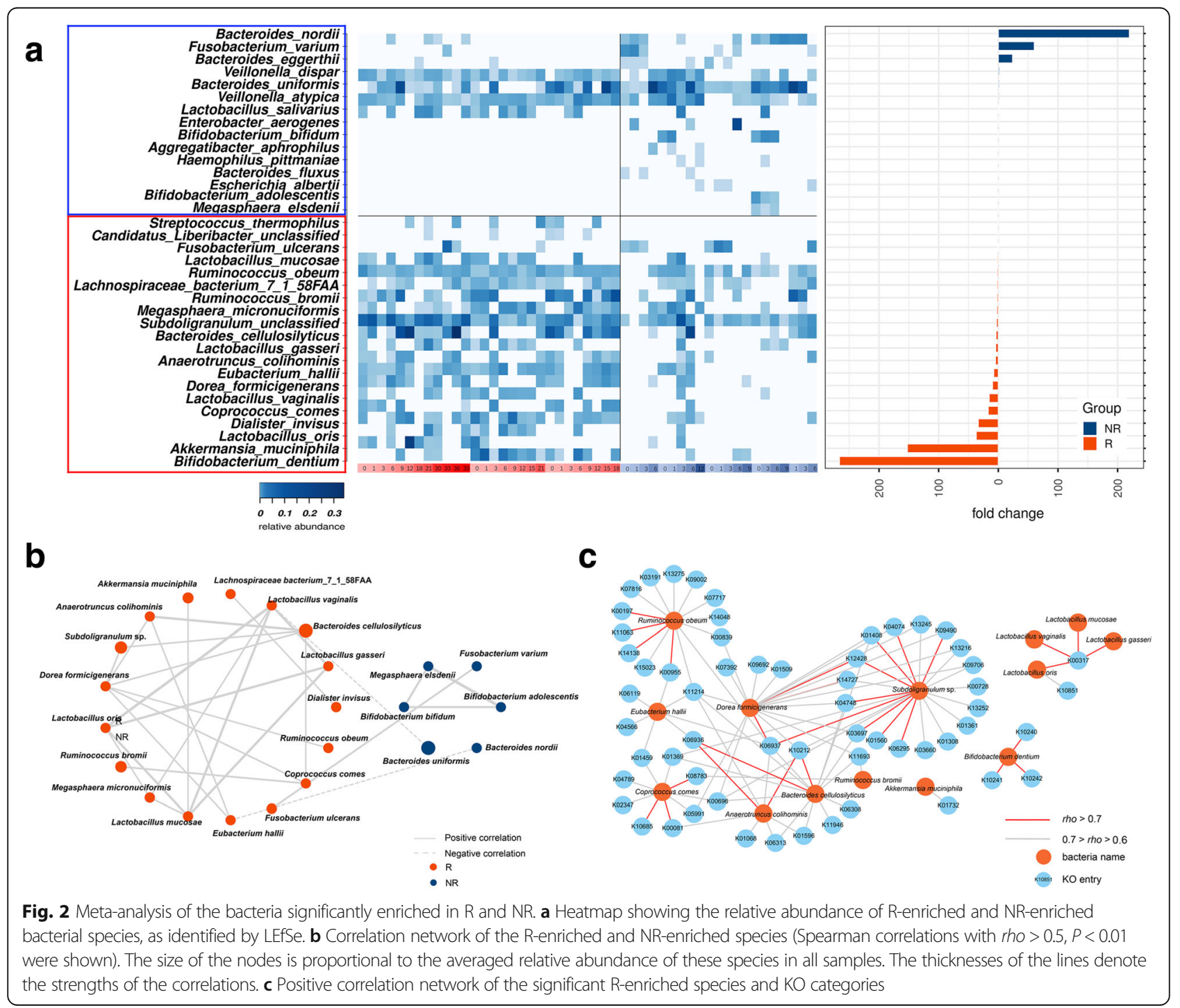


efficacy of anti-PD-1 immunotherapy [8]. In the present study, the SparCC algorithm was applied to gain insights into the mutualistic networks between R-enriched and NRenriched species (Fig. 2b). The number of significant positive-correlation pairs and the correlation strengths in R-enriched species were higher than those of NR-enriched species. Among the R-enriched bacteria, the four Lactobacillus species were most significantly correlated with each other, indicating their possible pivotal roles in the network. Our findings further indicated the biological significance of particular bacterial strains during anti-PD-1 immunotherapy in HCC and may provide support for the development of a gut-microbiome-modulation scheme in immunotherapy.

Next, functional gene families associated with Renriched bacteria were investigated. A total of 189 Kyoto Encyclopedia of Genes and Genomes (KEGG) orthologies (KOs) were identified to be enriched in $\mathrm{R}$ (KruskalWallis rank sum test, $P<0.05)$. Significant positive correlations were identified between 123 R-enriched KOs and 18 R-enriched species (Spearman's correlation, rho > 0.5, Additional file 6: Table S1). Pathway identification of KOs verified the potential bioactivities of R-enriched species (Fig. 2c and Additional file 5: Figure S5). In detail, the cellobiose-transport system (ko02010) was significantly correlated with $B$. dentium; the pectin lyase (K01732), which may be involved in pectin metabolism, was correlated with A. muciniphila. Both cellulose and pectin have been highlighted for their prebiotic and antiinflammatory potential as dietary fibers $[11,12]$. Methanogenesis pathway (ko00680) was found to be correlated with $R$. obeum and the four Lactobacillus species. Methane generated in human gastrointestinal tract has been reported to ameliorate oxidative stress injury and suppress the host inflammatory response [13]. Other pathways with potential benefits included sulfate reduction (ko00920) and carbon fixation (ko00720) functions that were correlated with $R$. obeum, carotenoid biosynthesis (ko00906) correlated with B. cellulosilyticus and A. colihominis, and unsaturated fatty acid metabolism (ko00590) associated with C. comes (Additional file 7: Table S2). Such findings further illustrated the potential underlying mechanisms of gut microbiome influencing the anti-PD-1 immunotherapy efficacy in HCC patients.

It is well recognized that factors including age, genetics, and diet may influence microbiome composition [14]. However, the long-term stability of the human gut microbiota has been demonstrated in previous studies. A study demonstrated that an individual's microbiota could be remarkably stable, with $60 \%$ of strains remaining over the course of five years; this finding highlighted that such stability and responsiveness to physiologic change confirmed the potential of the gut microbiota as a diagnostic tool and therapeutic target
[15]. In the present study, patients were enrolled with strict criteria to minimize potential dietary or geographical influences during the whole course of treatment, as well as to ensure that the dynamic changes of gut microbiota in both $R$ and NR were attributed to the therapeutic intervention instead of any daily factors.

Although our present microbiome-wide association study highlighted the association between changes in the gut microbiome and host-immune responses to drugs, any causal relationship of these correlative associations remains unknown. To further elucidate this relationship, several strategies have been developed. Firstly, 'metaomic' analysis combining sequencing and multiple biochemical techniques can greatly advance the knowledge of the human microbiome and its specific role in governing disease states. For example, Gopalakrishnan et al. compared the tumor-associated immune infiltrates via multi-parameter immunohistochemistry (IHC) and found a statistically significant positive correlation between CD8+ T cell infiltrate in the tumor and the Faecalibacterium genus as well as the Ruminococcaceae family, suggesting a possible mechanism through which the gut microbiome may modulate anti-tumor immune responses [7]. More importantly, in vitro or in vivo experimental models are required in order to allow the systematic manipulation of variables and, thus, allow experimental testing and validation of results derived from meta-omics [16]. In our previous study, we had demonstrated that Behcet's disease (BD), a kind of autoimmune disorder, was associated with considerable gut microbiome changes [17]. To determine whether the gut microbiome contributes to development of this disease, fecal microbiota transplantation (FMT) was performed in mice undergoing autoimmune uveitis. We demonstrated that mice colonized with whole-gut microbiomes from $\mathrm{BD}$ patients showed an exacerbation of disease activity and an excessive production of pro-inflammatory cytokines. These results may confirm the hypothesis that specific bacterial patterns contributed to the development of intraocular inflammatory disease. In our future studies, similar meta-omics strategies, as well as the use of mouse FMT models, will also be incorporated in order to deeply explore the interaction between the gut microbiome and host responses to immunotherapies among HCC patients.

Gopalakrishnan et al. also assessed the landscape of the oral and gut microbiomes in patients with metastatic melanoma receiving anti-PD-1 immunotherapy and demonstrated a high abundance of Lactobacillales in the oral microbiomes compared with that of fecal microbiomes in all subjects [7]. Galloway-Pena et al. further reported a high degree of intra-patient temporal instability of both stool and oral microbial diversity in patients with acute myeloid leukemia undergoing induction 
chemotherapy [18]. Both studies showed the possibility of using oral microbiome, rather than fecal microbiome, as an indicator for specific clinical outcomes. In our future longitudinal studies, both fecal and oral microbiome will be taken into consideration for a more comprehensive visualization of the host-microbe interactions.

In conclusion, by metagenomic sequencing of periodic fecal samples, we illustrated that the dynamic-variation characteristics of the gut microbiome might be used for early prediction of the six-month outcomes of anti-PD-1 immunotherapy in HCC at 3-6 weeks after treatment initiation, which is critical for disease monitoring and treatment decision-making. To our knowledge, this is the first study to focus on the association between the gut microbiome and the response to anti-PD-1 immunotherapy for HCC. Furthermore, while previous studies had mostly only provided cross-sectional comparisons, the present study showed the different trajectories of microbiome shifts between $\mathrm{R}$ and NR and revealed a stronger stability of the gut microbiome in $\mathrm{R}$ during the whole course of treatment. We believe that the potential of the gut microbiota as a therapeutic target is indicated in this study, and that the relevant bacterial species and metabolic pathways revealed here could be developed as a modulation strategy for better treatment options for HCC patients.

\section{Methods}

\section{Patients and medications}

A cohort of eight patients was included in this study. All patients were histologically confirmed HCC with BCLC Stage $\mathrm{C}$ disease and had experienced disease progression on first-line sorafenib treatment. Other eligibility criteria included radiological measurable disease, an adequate function of major organs, an Eastern Cooperative Oncology Group (ECOG) performance status of 0 or 1 , and a Child-Pugh Class A liver function. Exclusion criteria included fibrolamellar HCC, sarcomatoid HCC, or mixed cholangiocarcinoma and $\mathrm{HCC}$, liver transplantation, or a history of active autoimmune disease.

Patients received camrelizumab (SHR-1210, HengRui Medicine Co., Jiangsu, China), a humanized anti-PD-1 IgG4 monoclonal antibody, intravenously at a dose of 3 $\mathrm{mg} / \mathrm{kg}$ every three weeks until disease progression or intolerance (Clinicaltrials.gov ID: NCT02989922). Written informed consent, including collection and analysis of microbiome samples, was obtained from each patient before initiation of the study. Patients were asked to keep their own dietary and other habits during drug treatment to avoid any interruption to their inherent gut microbiome. Additionally, in order to omit the geographical influence, we collected samples only from one hospital with local individuals who shared common dietary intake patterns. None of the patients had experienced diarrhea or other intestinal symptoms, or antibiotic/probiotic consumption during the study.

Radiological evaluation was assessed every six weeks according to the RECIST 1.1 criteria. Responders (R, $n=3$ ) were defined by radiographic evidence as complete or partial response, or stable disease lasting for at least six months. Non-responders (NR, $n=5)$ were defined as those with progressed disease or stable disease lasting less than six months. This study complied with the Declaration of Helsinki and was approved by the Ethics Committee of the First Affiliated Hospital of Zhejiang University.

\section{Fecal sample collection}

Fresh feces were collected on the last pre-treatment clinic visit (as baseline, Day 0), one week after treatment initiation, the day of each treatment and were stored at $-80^{\circ} \mathrm{C}$ immediately prior to DNA extraction. According to the sample providing policy in the informed consent, the observation lasted for 39,21 , and 18 weeks for the three responders, and 6, 6, 9, 9, and 12 weeks for the five non-responders.

\section{DNA extraction and metagenomic sequencing}

Dynamic variation of gut bacterial composition of $R$ and NR during the anti-PD-1 immunotherapy was evaluated using fecal metagenomic sequencing. Briefly, bacterial genomic DNA was extracted using QIAamp DNA Stool Mini Kit (Qiagen, Hilden, Germany). After checking the integrity and concentration of DNA, individual libraries were constructed using MGIEasy DNA Library Prep Kit (BGI, Shenzhen, China), loaded into the BGISEQ-500 RS platform (BGI, Shenzhen, China), and were sequenced using $2 \times 100 \mathrm{bp}$ paired-end read protocol. The process of quality filtering, trimming, and demultiplexing was carried out as described previously [19]. A total of 49 datasets ( 28 from $\mathrm{R}$ and 21 from NR) were generated. Overall, $78.12 \%$ of the raw reads were regarded as highquality reads, with an average length of $72 \mathrm{bp}$ and an average Q35 score of 100\% (Additional file 8: Table S3).

\section{Taxonomic and gene profiling}

All high-quality reads were then aligned to Homo sapiens (human) genome assembly hg38 [20] using SOAPalign 2.21 with default parameters to remove human reads (https:// anaconda.org/bioconda/soapaligner). The retained clean reads were then aligned to $\sim 1 \mathrm{M}$ clade-specific marker genes from approximately 17,000 reference genomes for estimation of relative phylotype abundance using MetaPhlAn (version 2.5.0) [21].

For gene annotation, the clean reads were aligned to the integrated gene catalog (IGC) [22] by using SOAPalign 2.21 with default parameters; only the reads with 
both ends mapped to the same gene were used in the following analysis. An average IGC mapping rate of $77.77 \%$ and an average unique mapping rate of $63.27 \%$ were achieved. The gene relative abundance profile was generated following the procedure described before [17]. Functional annotations were carried out by BLASTP search against the KEGG database ( $e$ value $\leq 1 \mathrm{e}-5$ and high-scoring segment pair scoring > 60) [23]. The KO abundance was estimated by accumulating the relative abundance of all genes belonging to this feature.

\section{Statistical analyses and correlation network}

Non-parametric Wilcoxon rank-sum test was employed to analyze the statistical significance of diversity indices, taxa and KOs between R and NR. Bray-Curtis metrics were applied to calculate pairwise dissimilarities between samples and were used in beta diversity evaluation and principal coordinate analysis (PCoA) [24]. The LEfSe algorithm was applied to identify the phylotypes significantly different in relative abundance between all $\mathrm{R}$ and NR samples; phylotypes with an LDA score cut-off of 2.0 and $P<0.05$ in built-in rank sum test were regarded as statistically significant [25].

SparCC algorithm was used to calculate correlations between R-enriched and NR-enriched species. Bootstrapping of 100 repetitions was used to compute the $P$ value for each correlation, as described previously [17]. Only significant correlations with $P<0.05$ and rho $>0.5$ were presented in the network. Spearman correlation was applied to estimate the association strengths for determining the relationship between bacteria and $\mathrm{KO}$ categories. Only significant correlations with $P<0.01$ and $r h o>0.5$ were presented in the network. The speciesspecies network and the species-KO network were both visualized with Cytoscape3.0.2.

\section{Additional files}

Additional file 1: Figure S1. Principal componeLnt analysis (PCoA) based on Bray-Curtis distances, followed by Adonis test at Day 0 (a), Week 1 (b), Week 3 (c), and Week 6 (d). (PNG 1455 kb)

Additional file 2: Figure S2. Microbial composition of the R (left) and NR (right) at the genus (a) and species (b) levels. The ten most abundant genera or species of each group are shown. (PNG 4920 kb)

Additional file 3: Figure S3. Sankey analysis of all R and NR during the treatment. (PNG 9946 kb)

Additional file 4: Figure S4. Differentially abundant genera (a) and species (b) between R and NR, identified by LEfSe. (PNG 2863 kb)

Additional file 5: Figure S5. Positive correlation network of significant R-enriched species and KOs. Correlations with rho $>0.7$ are shown in red, while those with rho $>0.5$ are shown in gray. (PNG 3679 kb)

Additional file 6: Table S1. List of significant positive correlations between species and KOs. (XLSX $27 \mathrm{~kb}$ )

Additional file 7: Table S2. Pathway assignment of the most significantly correlated KOs in R. (XLSX 14 kb)
Additional file 8: Table S3. Quality control of the sequencing data. (XLSX $19 \mathrm{~kb})$

\section{Abbreviations}

BCLC: Barcelona Clinic Liver Cancer; ECOG: Eastern Cooperative Oncology Group; HCC: hepatocellular carcinoma; IGC: integrated gene catalog; KEGG: Kyoto Encyclopedia of Genes and Genomes; KOs: Kyoto Encyclopedia of Genes and Genomes Orthologies; LDA: Linear discriminant analysis; LEfSe: Linear discriminant analysis - effect size; NR: Non-responders; ORR: Objective response rate; PCoA: Principal coordinate analysis; PD1: Programmed cell death protein 1; PD-L1: Programmed cell death protein 1 ligand 1; R: Responders; RECIST 1.1: Response Evaluation Criteria in Solid Tumors 1.1

\section{Acknowledgements}

We would like to thank the patients for their participation. We are grateful to all the participants who have made this research possible.

\section{Authors' contributions}

YZ, TW, SC, NQ and WF designed the study. YZ, TW, XT, HZ, WJ, PZ and PL collected samples and completed the laboratory experiments. TW, YH, DT and RS performed the statistical analyses. NQ and WF led the study. YZ, TW, $X T$, SC and DT drafted the manuscript. All authors reviewed the manuscript, provided feedback, and approved the manuscript in its final form.

\section{Funding}

Dr. NQ was supported by the National Natural Science Foundation of China (31670118) and the Natural Science Foundation of Zhejiang Province (LR15H030002). Dr. WF was supported by the Key Subject of Clinical Medical Engineering of Zhejiang Province (No. G3221), S\&T Major Project of China (2017ZX10203205), and the Major Scientific Project of Zhejiang Province (2017C03028).

\section{Availability of data and materials}

All sequencing datasets were uploaded to the NCBI database with SRA accession: PRJNA505228.

\section{Ethics approval and consent to participate}

This study was approved by the Ethics Committee of the First Affiliated Hospital of Zhejiang University. All subjects provided informed consent to be included in the study.

\section{Consent for publication}

Not applicable.

\section{Competing interests}

The authors declare that they have no competing interests.

\section{Author details}

${ }^{1}$ Cancer Biotherapy Center, First Affiliated Hospital, School of Medicine, Zhejiang University, 79 Qingchun Road, Hangzhou, China. ${ }^{2}$ Realbio Genomics Institute, 138 Xinjunhuan Road, Shanghai, China. ${ }^{3}$ Shanghai Tenth People's Hospital Affiliated to Tongji University, No. 301 Yanchangzhong Road, Shanghai, China. ${ }^{4}$ Key Laboratory for Drug Evaluation and Clinical Research of Zhejiang Province, First Affiliated Hospital, School of Medicine, Zhejiang University, 79 Qingchun Road, Hangzhou, China. ${ }^{5}$ Key Laboratory of Precision Diagnosis and Treatment for Hepatobiliary and Pancreatic Tumor of Zhejiang Province, First Affiliated Hospital, School of Medicine, Zhejiang University, 79 Qingchun Road, Hangzhou, China. ${ }^{6}$ Zhejiang Provincial Key Laboratory of Pancreatic Disease, First Affiliated Hospital, School of Medicine, Zhejiang University, 79 Qingchun Road, Hangzhou, China.

Received: 26 February 2019 Accepted: 21 June 2019

Published online: 23 July 2019

\section{References}

1. Siegel RL, Miller KD, Jemal A. Cancer statistics, 2018. CA Cancer J Clin. 2018; 68(1):7-30.

2. Cheng AL, Kang YK, Chen Z, Tsao CJ, Qin S, Kim JS, et al. Efficacy and safety of sorafenib in patients in the Asia-Pacific region with advanced 
hepatocellular carcinoma: a phase III randomised, double-blind, placebocontrolled trial. The Lancet Oncology. 2009;10(1):25-34.

3. El-Khoueiry AB, Sangro B, Yau T, Crocenzi TS, Kudo M, Hsu C, et al. Nivolumab in patients with advanced hepatocellular carcinoma (CheckMate 040): an open-label, non-comparative, phase 1/2 dose escalation and expansion trial. Lancet (London, England). 2017; 389(10088):2492-502

4. Zhu AX, Finn RS, Edeline J, Cattan S, Ogasawara S, Palmer D, et al. Pembrolizumab in patients with advanced hepatocellular carcinoma previously treated with sorafenib (KEYNOTE-224): a non-randomised, open-label phase 2 trial. The Lancet Oncology. 2018;19(7):940-52.

5. Matson V, Fessler J, Bao R, Chongsuwat T, Zha Y, Alegre ML, et al. The commensal microbiome is associated with anti-PD-1 efficacy in metastatic melanoma patients. Science. 2018;359(6371):104.

6. Vetizou M, Pitt JM, Daillere R, Lepage P, Waldschmitt N, Flament C, et al. Anticancer immunotherapy by CTLA-4 blockade relies on the gut microbiota. Science. 2015; 350(6264):1079-84.

7. Gopalakrishnan V, Spencer CN, Nezi L, Reuben A, Andrews MC, Karpinets TV, et al. Gut microbiome modulates response to anti-PD-1 immunotherapy in melanoma patients. Science. 2018;359(6371):97-103.

8. Routy B, Le Chatelier E, Derosa L, Duong CPM, Alou MT, Daillere R, et al. Gut microbiome influences efficacy of PD-1-based immunotherapy against epithelial tumors. Science. 2018;359(6371):91-7.

9. Human Microbiome Project C. Structure, function and diversity of the healthy human microbiome. Nature. 2012:486(7402):207-14.

10. Sivan A, Corrales L, Hubert N, Williams JB, Aquino-Michaels K, Earley ZM, et al. Commensal Bifidobacterium promotes antitumor immunity and facilitates anti-PDL1 efficacy. Science. 2015;350(6264):1084-9.

11. Shoaie S, Ghaffari P, Kovatcheva-Datchary P, Mardinoglu A, Sen P, Pujos-Guillot $E$, et al. Quantifying diet-induced metabolic changes of the Human gut Microbiome. Cell Metab. 2015;22(2):320-31.

12. Chung WSF, Meijerink M, Zeuner B, Holck J, Louis P, Meyer AS, et al. Prebiotic potential of pectin and pectic oligosaccharides to promote anti-inflammatory commensal bacteria in the human colon. FEMS Microbiol Ecol. 2017;1, 93(11).

13. Zhang $X$, Li N, Shao H, Meng Y, Wang L, Wu Q, et al. Methane limit LPS-induced NF-kappaB/MAPKs signal in macrophages and suppress immune response in mice by enhancing PI3K/AKT/GSK-3betamediated IL-10 expression. Sci Rep. 2016;6:29359.

14. Wu GD, Chen J, Hoffmann C, Bittinger K, Chen YY, Keilbaugh SA, et al. Linking long-term dietary patterns with gut microbial enterotypes. Science. 2011;334(6052):105-8.

15. Faith JJ, Guruge JL, Charbonneau M, Subramanian S, Seedorf H, Goodman AL, et al. The long-term stability of the human gut microbiota. Science. 2013;341(6141):1237439.

16. Fritz JV, Desai MS, Shah P, Schneider JG, Wilmes P. From meta-omics to causality: experimental models for human microbiome research. Microbiome. 2013;1(1):14

17. Ye Z, Zhang N, Wu C, Zhang X, Wang Q, Huang X, et al. A metagenomic study of the gut microbiome in Behcet's disease. Microbiome. 2018:6(1):135.

18. Galloway-Pena JR, Smith DP, Sahasrabhojane P, Wadsworth WD, Fellman BM, Ajami NJ, et al. Characterization of oral and gut microbiome temporal variability in hospitalized cancer patients. Genome medicine. 2017;9(1):21.

19. Fang $C$, Zhong H, Lin $Y$, Chen $B$, Han $M$, Ren $H$, et al. Assessment of the CPAS-based BGISEQ-500 platform for metagenomic sequencing. GigaScience. 2018;7(3):1-8.

20. Rosenbloom KR, Armstrong J, Barber GP, Casper J, Clawson H, Diekhans M, et al. The UCSC genome browser database: 2015 update. Nucleic Acids Res. 2015;43(Database issue):D670-81.

21. Truong DT, Franzosa EA, Tickle TL, Scholz M, Weingart G, Pasolli E, et al. MetaPhIAn2 for enhanced metagenomic taxonomic profiling. Nat Methods. 2015;12(10):902-3.

22. Li J, Jia $H$, Cai $X$, Zhong $H$, Feng $Q$, Sunagawa $S$, et al. An integrated catalog of reference genes in the human gut microbiome. Nat Biotechnol. 2014;32(8):834-41.

23. Kanehisa M, Goto S, Kawashima S, Okuno Y, Hattori M. The KEGG resource for deciphering the genome. Nucleic Acids Res. 2004; 32(Database issue):D277-80.
24. Parks DH, Beiko RG. Measures of phylogenetic differentiation provide robust and complementary insights into microbial communities. The ISME Journal. 2013;7:173-83.

25. Segata N, Izard J, Waldron L, Gevers D, Miropolsky L, Garrett WS, et al. Metagenomic biomarker discovery and explanation. Genome Biol. 2011;12(6):R60

\section{Publisher's Note}

Springer Nature remains neutral with regard to jurisdictional claims in published maps and institutional affiliations.
Ready to submit your research? Choose BMC and benefit from:

- fast, convenient online submission

- thorough peer review by experienced researchers in your field

- rapid publication on acceptance

- support for research data, including large and complex data types

- gold Open Access which fosters wider collaboration and increased citations

- maximum visibility for your research: over $100 \mathrm{M}$ website views per year

At $\mathrm{BMC}$, research is always in progress.

Learn more biomedcentral.com/submissions 